\title{
The role of microscope-integrated near-infrared indocyanine green videoangiography in the surgical treatment of intracranial dural arteriovenous fistulas
}

\author{
Harjot Thind, MD, MPH, Douglas A. Hardesty, MD, Joseph M. Zabramski, MD, \\ Robert F. Spetzler, MD, and Peter Nakaji, MD
}

Division of Neurological Surgery, Barrow Neurological Institute, St. Joseph's Hospital and Medical Center, Phoenix, Arizona

OBJECT The successful treatment of an intracranial dural arteriovenous fistula (dAVF) requires complete obliteration of blood flow through the fistulous point. Surgical ligation is often used along with endovascular techniques. Digital subtraction angiography (DSA) can be used to confirm fistula obliteration; however, this technique can be cumbersome intraoperatively and difficult to correlate anatomically with the surgical field. Near-infrared indocyanine green (ICG) videoangiography has been described as a complementary tool for this purpose.

METHODS The authors examined intracranial dAVF cases in which microscope-integrated intraoperative ICG videoangiography was used to identify and/or confirm obliteration of the dAVF during surgery. Retrospective evaluation of all intracranial dAVF cases treated with surgical ligation over a 10-year period at the Barrow Neurological Institute $(n=47)$ revealed 28 cases in which ICG videoangiography was used. The results were compared with findings on preoperative and intraoperative or postoperative DSA.

RESULTS ICG videoangiography successfully confirmed the fistulous point intraoperatively in $96 \%$ (22/23) of the cases. It also revealed complete obliteration of fistulas, comparable to intraoperative or postoperative DSA, in 91\% (21/23) of the cases. The false-negative rate of ICG was $8.7 \%(2 / 23)$, which is similar to the false-negative rate of intraoperative DSA alone $(10.5 \%[2 / 19])$

CONCLUSIONS Microscope-based ICG videoangiography provides real-time information about the intraoperative anatomy of dAVFs. In addition, it can confirm complete obliteration of a fistula. This technique may be useful during dAVF surgery as an independent form of angiography or as an adjunct to intraoperative or postoperative DSA.

http://thejns.org/doi/abs/10.3171/2014.11.JNS14947

KEY WORDS indocyanine green videoangiography; intracranial dural arteriovenous fistula; intraoperative imaging techniques; vascular disorders

$\mathrm{D}$ URAL arteriovenous fistulas (dAVFs) account for $10 \%-15 \%$ of intracranial vascular malformations. ${ }^{10}$ Although some fistulas thrombose spontaneously, their pathological course usually progresses from venous sinus hypertension to occlusion to retrograde cortical venous drainage. Fistulas may be an incidental finding, or patients may suffer a wide range of symptoms from mild headache to visual deficits to fatal hemorrhage.

Surgery, embolization, radiation therapy, or combinations thereof have been used to treat dAVFs. However, surgical intervention remains the treatment of choice in many cases. Surgical treatment of dAVFs has evolved from disconnection of the fistula and resection of the involved segment of dura and venous sinus to surgical disconnection of the venous drainage alone. The latter procedure has proved to be as efficacious as resection with less risk of venous stroke. 3,6

Preoperative imaging, such as MRI, CT angiography, and digital subtraction angiography (DSA), is essential for determining the location of, and the surgical approach to, dAVFs. Intraoperatively, the point at which the fistula transitions from the arterial to the venous component must be identified, and DSA can be used for this purpose. However, DSA has significant disadvantages; it requires an angiographer, a significant investment of time, radiation exposure, and the interruption of surgery. Furthermore,

ABBREVIATIONS dAVF = dural arteriovenous fistula; $D S A=$ digital subtraction angiography; $I C G$ = near-infrared indocyanine green .

SUBMITTED April 28, 2014. ACCEPTED November 11, 2014.

INCLUDE WHEN CITING Published online January 2, 2015; DOI: 10.3171/2014.11.JNS14947.

DISCLOSURE The authors report no conflict of interest concerning the materials or methods used in this study or the findings specified in this paper. 
substantial experience is required to correlate correctly the vessels seen on the angiogram with those seen within the surgical field.

We now use microscope-integrated near-infrared indocyanine green (ICG) videoangiography to examine dAVFs intraoperatively before and after open surgical obliteration. This ICG videoangiography technique allows real-time intraoperative visualization of vascular flow via an integrated near-infrared excitation source and camera integrated into the surgical microscope after a systemic intravenous injection of ICG. This technique has already proven useful in cerebral aneurysm surgery, extracranial-intracranial bypass, and arteriovenous malformation treatment as an independent form of angiography or as an adjunct to intraoperative or postoperative DSA. ${ }^{5,8,12,13,15} \mathrm{In}$ the present study, we retrospectively evaluated our experience with ICG videoangiography in identifying patients treated for intracranial dAVFs and in documenting the rate of complete obliteration of the dAVF at the time of surgery compared with preoperative and intraoperative or postoperative DSA.

\section{Methods}

This study was approved by the St. Joseph's Hospital and Medical Center Institutional Review Board for Human Research. Intracranial dAVF cases that were treated via open surgical obliteration at Barrow Neurological Institute between January 2003 and December 2012 were identified retrospectively. During this time period, there were intervals in which ICG was unavailable because of supply interruption. The use of ICG videoangiography was left to the discretion of the operating neurosurgeon. The technical protocol of ICG videoangiography use in neurosurgical procedures has been described elsewhere. ${ }^{11}$ In brief, patients receive an intravenous injection of ICG. The dye reaches the cerebral vasculature within 30 seconds and is visualized using an OPMI Pentero Microscope (Carl Zeiss Co.) that has an integrated near-infrared light source and a video camera for illumination and visualization purposes, respectively. ICG videoangiography provided qualitative information about the fistula before ligation. On the basis of the timing and direction of flow, both the early-filling fistula and the presence of abnormal retrograde flow were visualized. Postobliteration ICG videoangiography identified the cessation of abnormal drainage in the surrounding normal circulation and the patency of normal vessels.

The surgical procedures to occlude dAVFs included either clipping or coagulation and division of draining veins after open craniotomy and standard microneurosurgical approaches. The primary surgical strategy was surgical disconnection of the venous component. Only when this strategy was inadequate was the surrounding dura or dural venous sinus resected.

\section{Results}

We identified 47 patients who underwent open surgical intracranial dAVF obliteration between January 2003 and December 2012. Of these patients, 28 underwent ICG videoangiography with or without intraoperative DSA, and 19 underwent intraoperative DSA only (Table 1). The angiographic appearance of the dAVFs was classified using the
TABLE 1. Patient demographics

\begin{tabular}{lcc}
\hline \multicolumn{1}{c}{ Parameter } & ICG Group & DSA Group \\
\hline No. of patients & 28 & 19 \\
\hline Average (range) age in yrs & $52.6(17-79)$ & $49.9(1-77)$ \\
\hline M/F ratio & $21 / 7$ & $14 / 5$ \\
\hline $\begin{array}{l}\text { Previous attempts at treatment } \\
\text { (no. [\%]) }\end{array}$ & $12(43)$ & $13(68)$ \\
\hline $\begin{array}{l}\text { No. of intraop ICG runs } \\
\text { No. of intraop DSA runs }\end{array}$ & 50 & 0 \\
\hline Cognard-Merland type (no. [\%]) & 14 & 21 \\
\hline Unknown & $1(3.6)$ & $1(5.3)$ \\
\hline I & $1(3.6)$ & $1(5.3)$ \\
\hline IIA \& IIB & $8(28.6)$ & $7(36.8)$ \\
\hline IIA & $5(17.9)$ & $3(15.8)$ \\
\hline IIB & $7(25.0)$ & $4(21.1)$ \\
\hline III & $5(17.9)$ & $2(10.5)$ \\
\hline IV & $1(3.6)$ & $1(5.3)$ \\
\hline
\end{tabular}

* Values total $>100 \%$ due to rounding.

previously described Cognard-Merland system in all patients who underwent preoperative DSA. ${ }^{2}$ The majority of the patients were middle-aged men. Slightly fewer patients who underwent ICG videoangiography than those who underwent intraoperative DSA only had undergone previous attempts at fistula obliteration, although this difference was not statistically significant (43\% [12/43] vs 68\% [13/19]; p $=0.08$, Fisher exact test). Twelve patients $(43 \%)$ in the ICG group and $10(53 \%)$ in the DSA group $(\mathrm{p}>0.05)$ presented with acute intracranial hemorrhage from dAVF rupture before undergoing urgent surgery. Other presenting symptoms included tinnitus, headache, and altered mental status (Table 2), and these symptoms did not differ significantly between the ICG and DSA groups (all $p>0.05$ ). No patient in either group had secondary postoperative hemorrhage.

Twenty-eight patients underwent a total of 50 ICG videoangiography runs during surgery. Both preobliteration and postobliteration runs were performed in 18 of the 28 cases. For the 10 cases in which ICG videoangiography was not used both before and after obliteration, preobliteration ICG alone was used in 5 cases and postobliteration ICG alone was used in the other 5 cases. These 28 patients also underwent standard intraoperative $(n=13)$

TABLE 2. Presenting symptoms of 47 study patients*

\begin{tabular}{lcc}
\hline & \multicolumn{2}{c}{ No. of Cases (\%) } \\
\cline { 2 - 3 } Presenting Symptom(s) & ICG Group $(\mathrm{n}=28)$ & DSA Group $(\mathrm{n}=19)$ \\
\hline Headache & $14(50)$ & $9(47)$ \\
\hline Acute cerebral hemorrhage & $12(43)$ & $10(53)$ \\
\hline $\begin{array}{l}\text { Other (vertigo, cranial } \\
\text { nerve palsies, etc.) }\end{array}$ & $9(32)$ & $5(26)$ \\
\hline Altered mental status & $6(21)$ & $4(21)$ \\
\hline None & $3(11)$ & $0(0)$ \\
\hline Pulsatile tinnitus & $2(7)$ & $1(5.3)$ \\
\hline
\end{tabular}

* Patients may have had more than 1 presenting symptom; therefore, the percentages do not summate to $100 \%$. 
or postoperative ( $n=14)$ DSA except for 1 case, in which the patient underwent postoperative MRI/angiography. In no cases were adverse effects associated with the administration of ICG dye. No complications attributable to ICG videoangiography were encountered.

Nineteen patients in this period underwent a total of 21 intraoperative DSA runs during surgery without the use of ICG videoangiography. In all these cases, intraoperative DSA was performed only after surgical ligation of the fistula. Every patient underwent additional formal postoperative DSA $0-3$ months postoperatively. No patient had a complication attributable to intraoperative DSA.

\section{ICG Videoangiography Before Obliteration}

Preobliteration ICG videoangiography confirmed the presence of the dAVF in $22(96 \%)$ of the 23 cases in which it was used. In the remaining case, ICG videoangiography did not identify the dAVF because the fistula was somewhat remote from the site of hemorrhage and was therefore outside the exposed surgical field.

\section{ICG Videoangiography After Obliteration}

ICG videoangiography was performed after surgical interruption in 23 cases. No abnormal arterialized flow was seen on ICG videoangiography in any of these cases after obliteration.

In 21 (91\%) of 23 cases, intraoperative or postoperative DSA and MRI/angiography confirmed the intraoperative postligation ICG videoangiography findings of complete fistula obliteration. In the remaining 2 cases, ICG videoangiography missed residual fistulous connections that were identified on formal postoperative DSA (false-negative rate $8.7 \%$ ). Neither of these 2 patients had undergone intraoperative DSA. In 1 case, the residual dAVF was not considered significant enough to warrant additional treatment and was found to have spontaneously thrombosed at 3-month follow-up DSA. In the other case, the patient was lost to follow-up. No retrograde cortical venous drainage was identified in either case of residual fistula.

\section{Neurological Outcomes of Patients Who Underwent ICG Videoangiography}

Neurological status after surgery improved or remained stable in $21(75 \%)$ of the 28 patients. Two patients, despite aggressive treatment, declined neurologically after surgery. One patient's condition was complicated by postoperative cerebral edema and arterial and venous infarcts, and her family chose to withdraw care. The second patient developed an expanding thrombosed varix and a venous sinus thrombosis 2 days after surgery. Despite surgical decompression and endovascular thrombectomy, his condition declined further, and he was eventually declared brain dead.

The level of improvement or decline in postoperative neurological status was unknown in 5 cases in which the patients were lost to follow-up after hospitalization.

\section{Illustrative Case}

A 30-year-old man suffered brief acute neck pain 15 months before admission. He thereafter noticed intermittent shocklike sensations with neck flexion (the Lhermitte sign). He progressed to develop lower-extremity weakness followed by bowel incontinence and difficulty initiating micturition. Examination revealed the patient to have increased muscle tone and hyperreflexia, especially in the lower extremities.

Imaging of the brain and cervical spine (Figs. 1 and 2) showed serpiginous flow voids within the posterior fossa and rostral cervical spine. DSA showed a posterior fossa dAVF fed by both posterior meningeal arteries (Fig. 3).

Preoperative embolization was performed through the right posterior meningeal artery. Embolization through the left posterior meningeal artery was unsuccessful because of vessel size and tortuosity.

One day after embolization, a combined suboccipital, left far-lateral approach was taken to surgically interrupt the dAVF. Abnormal dilated vessels were immediately visualized after dural opening. ICG videoangiography performed at this point showed the draining vein, venous aneurysm, and dural feeders that were feeding the fistula through the dura (Fig. 4). An aneurysm clip was placed at the junction between the venous aneurysm and feeders within the dura to obliterate the fistula. ICG videoangiography was repeated and showed no further drainage through the abnormal veins (Fig. 5). Postoperative DSA (Fig. 6) and MRI (Figs. 7 and 8) confirmed obliteration of the dAVF. The patient's postoperative hospital course was unremarkable.

At follow-up examination, the patient reported that his

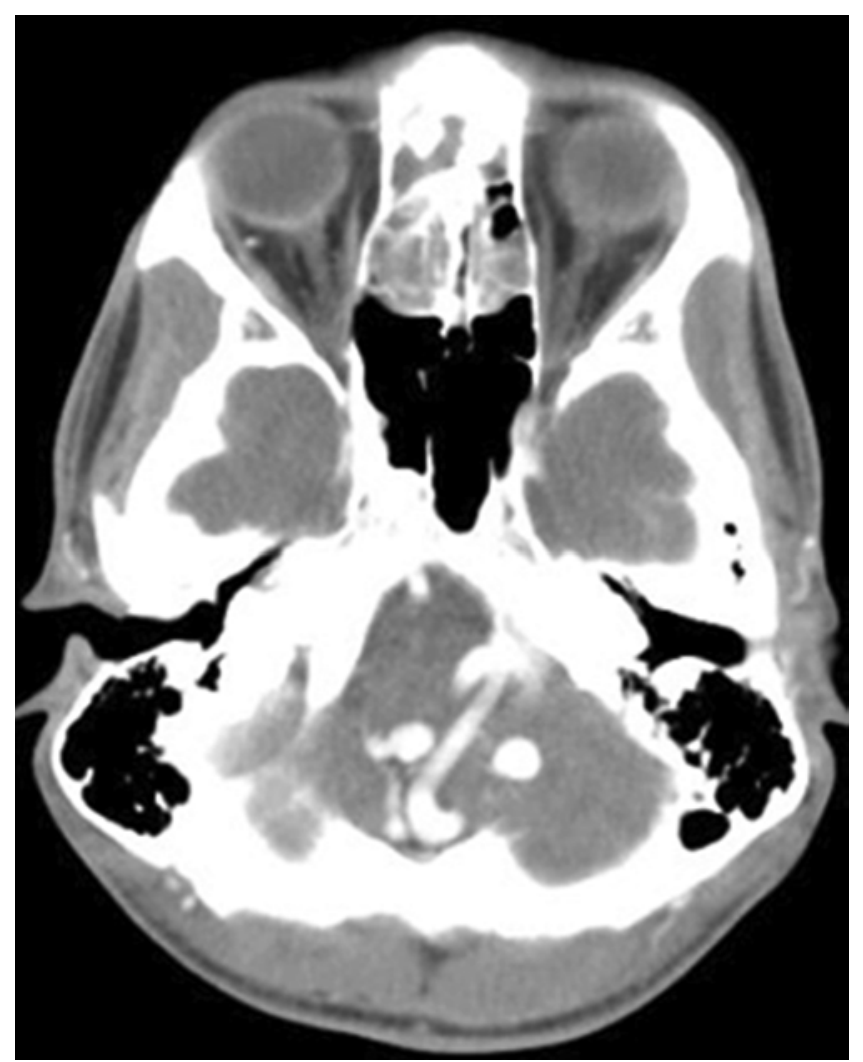

FIG. 1. Axial CT angiogram of the brain through the posterior fossa showing large dilated vessels. 


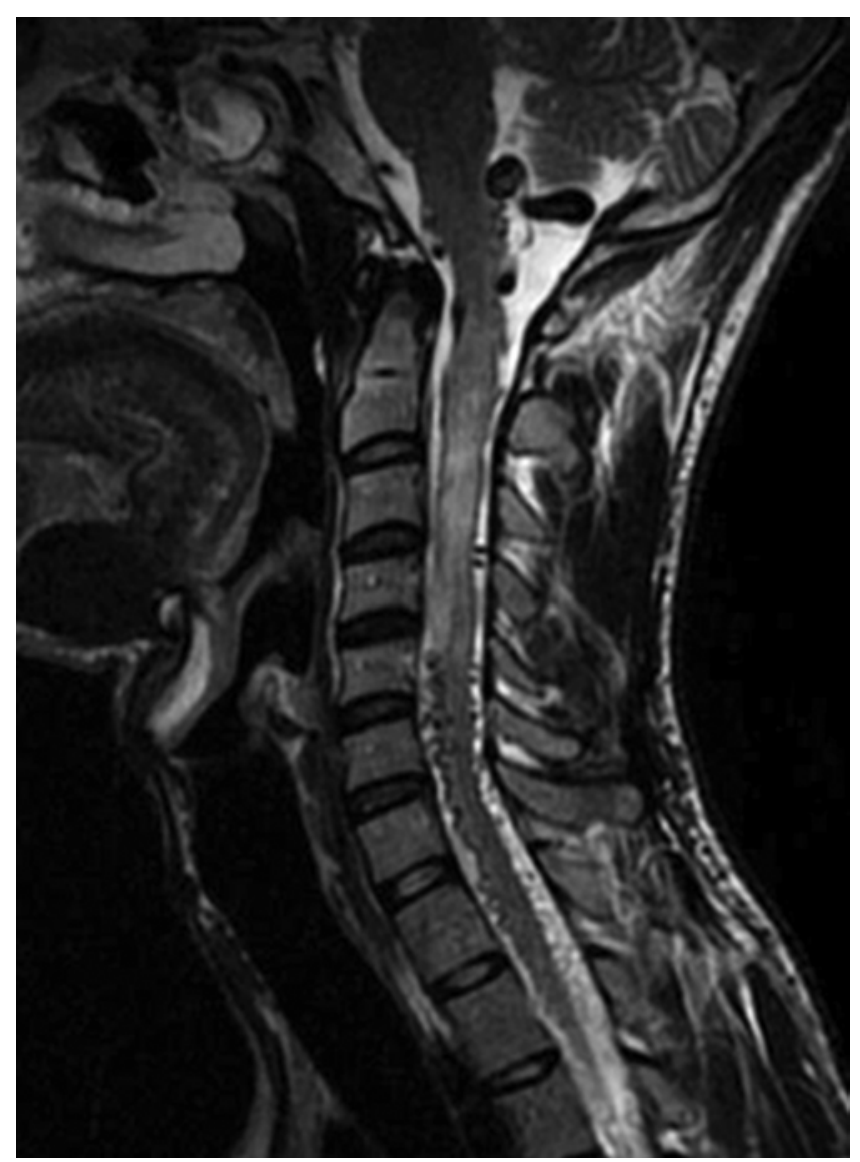

FIG. 2. Sagittal T2-weighted preoperative MR image showing edema in the cervical spinal cord, where dilated vessels cover the surface.

bowel and bladder functions were normalizing. His spasticity, however, had not improved significantly.

\section{Discussion}

Even with recent technical advances in the endovascular treatment of dAVFs, many cases can be treated easily and safely by surgical disconnection of the venous drainage. The principal challenges associated with the surgical approach are accurate intraoperative identification of the lesion and confirmation of its obliteration.

For preoperative detection, DSA remains the gold standard for the diagnosis of dAVFs. Intraoperative DSA is helpful, but it is invasive, involves ionizing radiation, and has a complication rate of $1 \%-3 \%$; possible complications include limb ischemia, retroperitoneal hematoma, and permanent neurological injury. ${ }^{4}$ Furthermore, correlating the angiographic anatomy with intraoperative findings can be difficult, even with considerable neurosurgeon experience. For dAVFs, in particular, distinguishing the draining venous complex from normal veins during surgery by observation alone is imprecise because even normal veins may appear engorged as a result of venous hypertension. CT angiography and MRI/angiography-based stereotactic navigation may help localize small, superficially located $\mathrm{dAVFs}$ but are not useful for identifying the fistulous transition.

Microscope-integrated ICG videoangiography has been

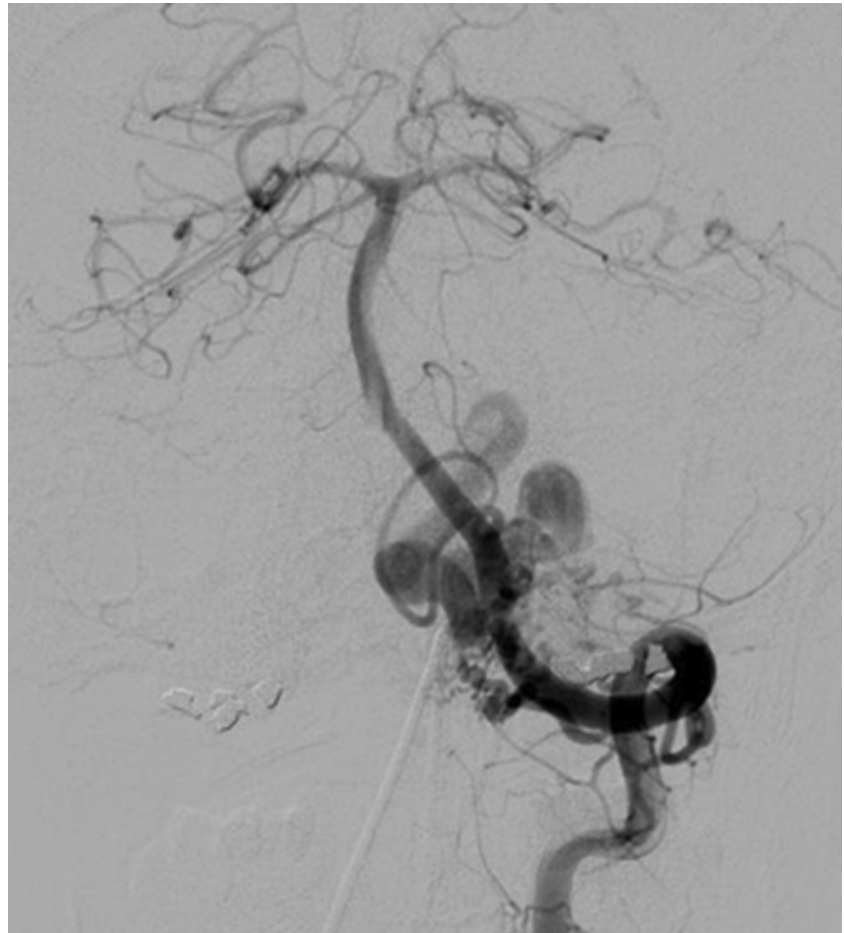

FIG. 3. Left vertebral artery injection angiogram (Towne projection), anteroposterior view, demonstrating a dilated vessel fed by branches coming off the left vertebral artery, with reflux into the posterior fossa

shown to be a useful adjunct, and possibly even an alternative, to DSA in several other vascular neurosurgical procedures. ${ }^{5,8,12,13,15}$ It provides real-time information about the patency, and direction of flow in, vessels of all sizes. ICG

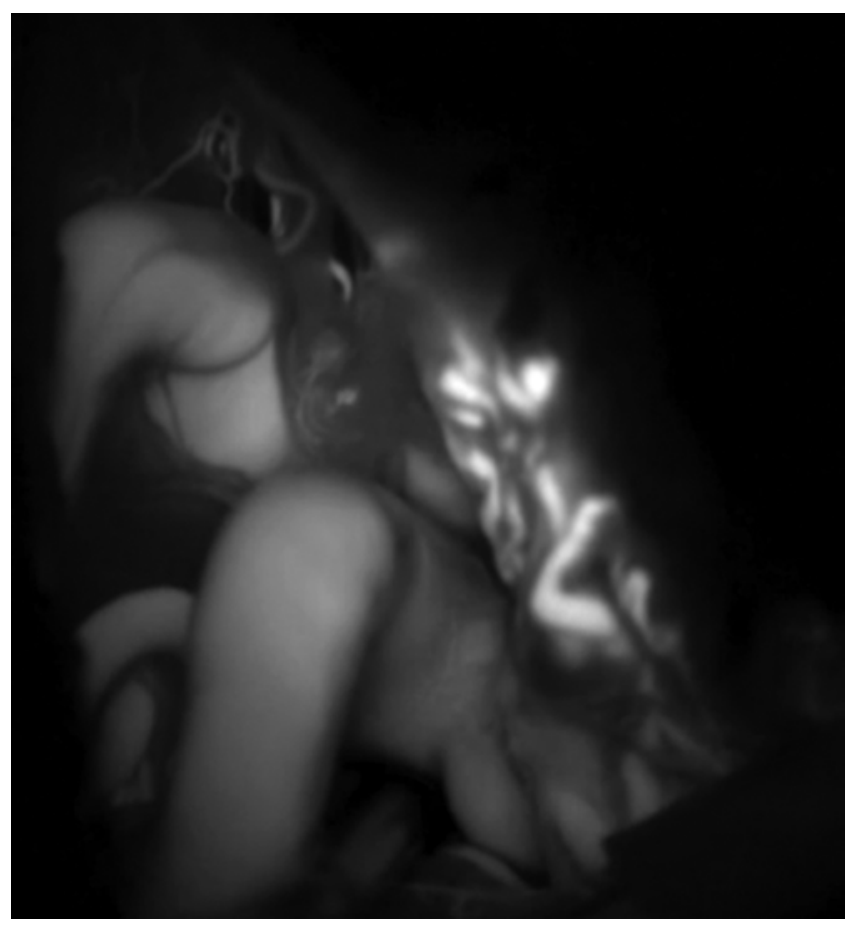

FIG. 4. Preobliteration intraoperative ICG videoangiogram showing early drainage of blood through 2 arterialized veins. 


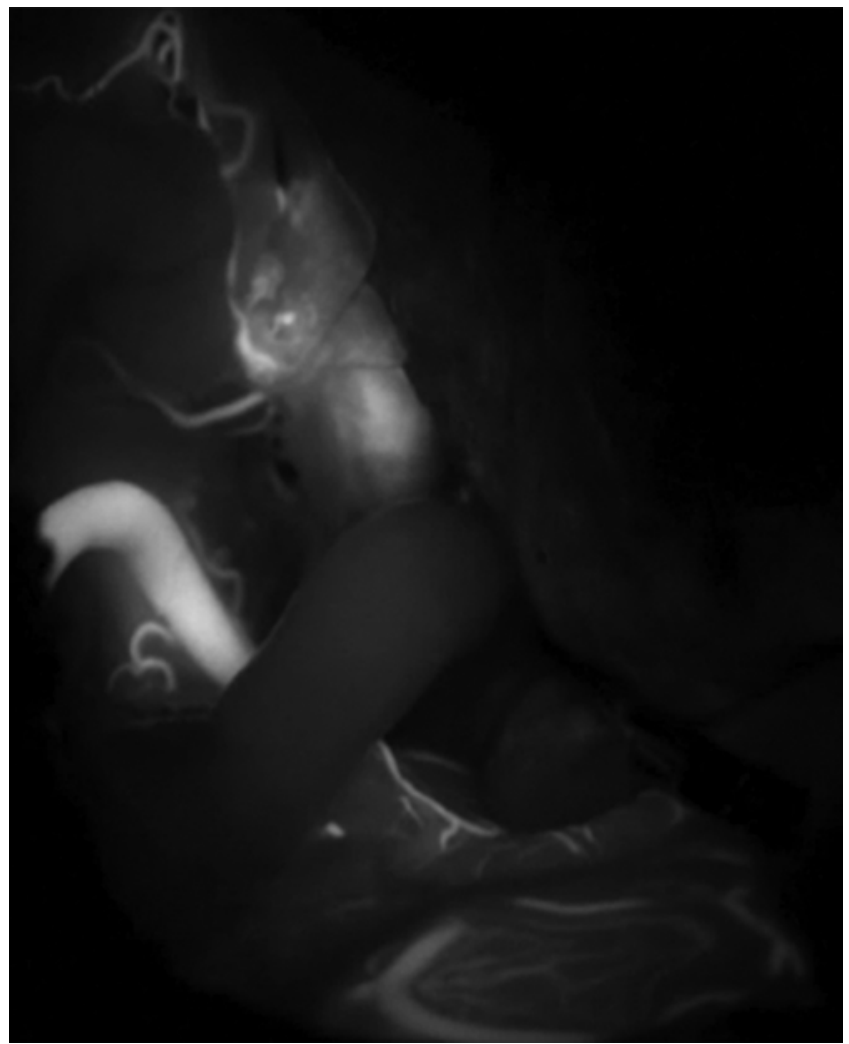

FIG. 5. Postclipping intraoperative ICG videoangiogram demonstrating no flow through the previously identified fistulous veins.

videoangiography has been used successfully in the surgical treatment of spinal dAVFs, ${ }^{1,14}$ even in angiographically occult cases. ${ }^{9}$ Despite these reports, there is a paucity of published data on ICG videoangiography usage in intracranial dAVFs, with the largest reported series consisting of only 13 cases,${ }^{14}$ the majority of which were incidental findings. In that study, intraoperative ICG matched other intraoperative and postoperative imaging in all 13 cases. ${ }^{14}$

We have presented here a retrospective review of 47 surgically treated intracranial dAVFs in which we used microscope-integrated ICG videoangiography in 28 cases. ICG videoangiography before surgical obliteration identified $96 \%$ of dAVFs, and postobliteration ICG videoangiography correctly identified complete disconnection of dAVFs in $91 \%$ of the cases.

Because our study was retrospective in nature, we cannot rule out an undetected bias in those patients who underwent ICG videoangiography versus those who underwent only intraoperative DSA without the use of ICG $(n=19)$. However, we found no statistically significant difference between these 2 groups with regard to CognardMerland type of fistula, preoperative or postoperative neurological status, hemorrhage, or previous attempts at dAVF treatment.

There are several advantages of intraoperative ICG videoangiography over intraoperative DSA. Unlike DSA, ICG videoangiography does not expose patients to ionizing radiation, and it takes less than 5 minutes to complete. A single intraoperative DSA run takes at least 30-45 minutes to complete. Accordingly, ICG videoangiography is

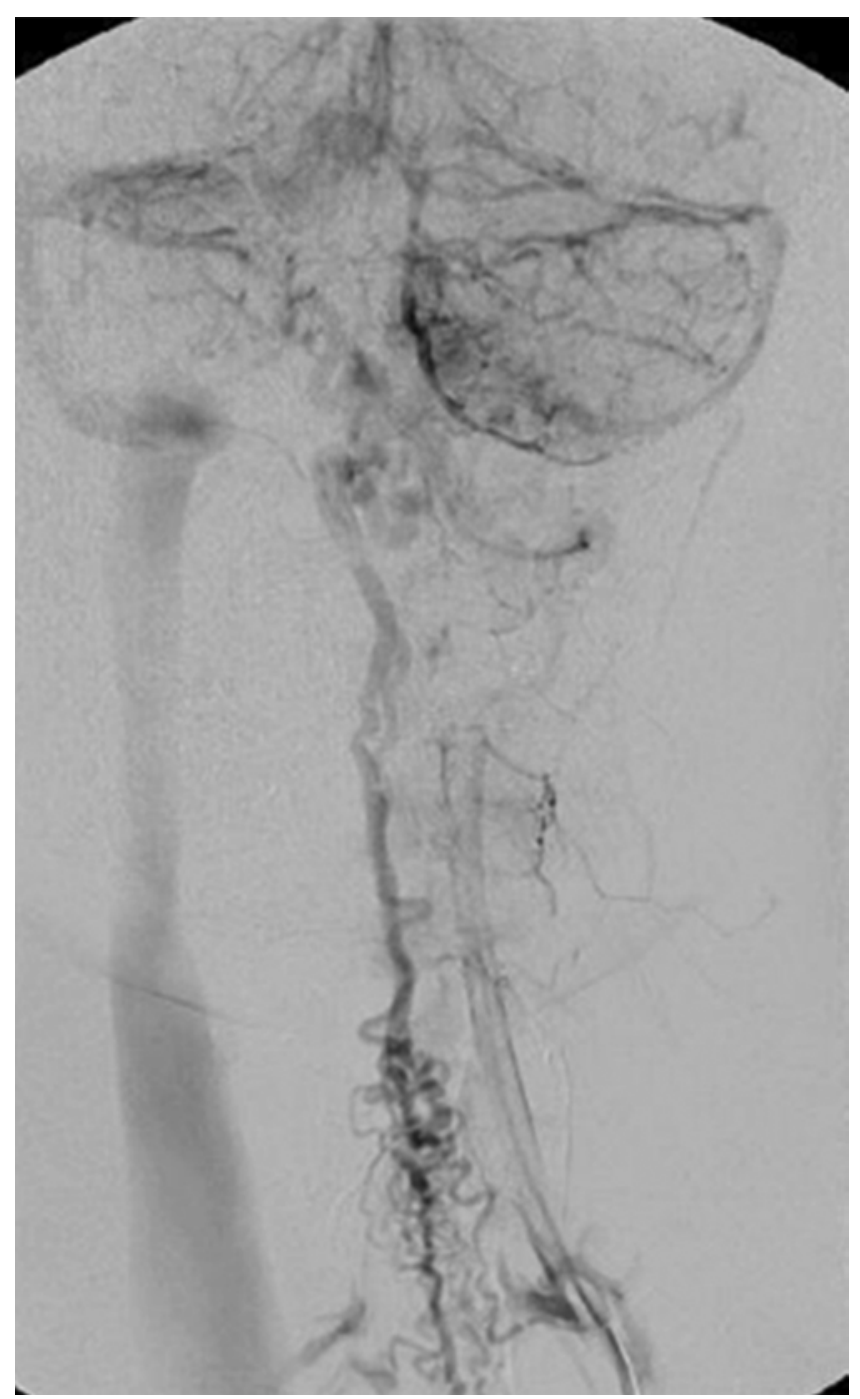

FIG. 6. Postoperative angiogram showing no evidence of early drainage or retrograde flow.

significantly less costly than intraoperative DSA with regard to operating room time. Furthermore, the results of ICG videoangiography are seen immediately within the surgical field rather than requiring extrapolation, as is the case with DSA. In our series, we did not encounter any adverse effects from the injection of ICG. In addition, this and other studies show that ICG videoangiography can be used safely multiple times during the same surgery?

However, as with every technique, there are limitations. The main drawback of ICG videoangiography is that visualization is restricted to the surgical field. Therefore, any filling vessels that are outside the field or obscured by bone, dura, or brain parenchyma will not be seen. This can be overcome somewhat by using multiple ICG videoangiography runs while moving the microscope and dynamically retracting portions of the surgical field. In addition, for optimal visualization, the field must be free of any blood/clots, debris, and calcifications. In our series, postobliteration ICG angiography missed 2 cases in which residual filling vessels (not associated with retrograde cortical drainage) were seen on postoperative DSA (false- 


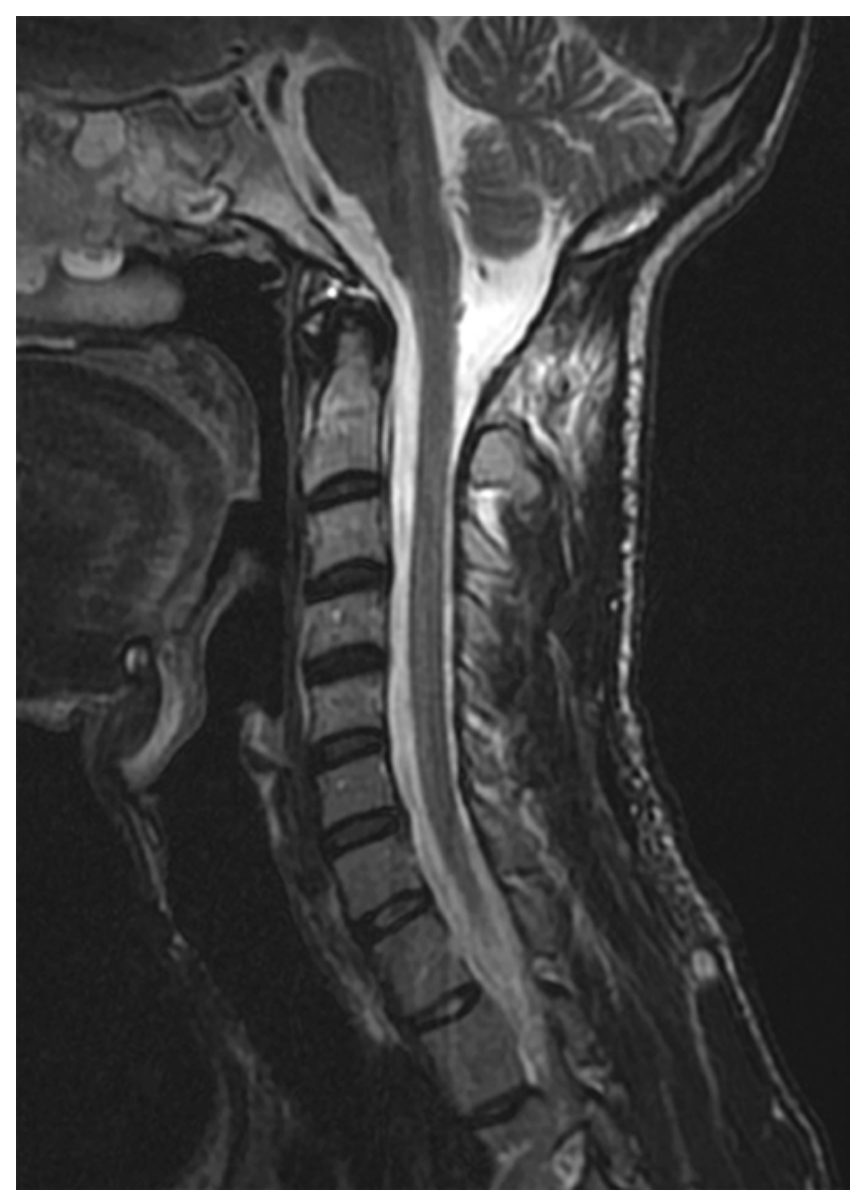

FIG. 7. Sagittal T2-weighted postoperative MR image demonstrating resolution of edema within the upper cervical spinal cord and resolution of previously seen cord signal change.

negative rate $8.7 \%$ ). However, those 2 cases represent the comparison of a simple, fast, intraoperative tool (ICG videoangiography) with the gold standard of formal biplane postoperative DSA. A better comparison is between the 2 intraoperative modalities of ICG videoangiography and intraoperative DSA. Comparison with postoperative DSA has revealed subtle findings that were missed by intraoperative DSA. In our 19 patients who received only intraoperative DSA, 2 patients were found to have residual filling on postoperative DSA that was not previously visualized (false-negative rate $10.5 \%$ ). Therefore, we believe that both intraoperative modalities have limitations, and there remains a place for formal postoperative angiography in demonstrating subtle dAVF filling. This makes ICG videoangiography even more attractive than intraoperative DSA, because it reduces the overall radiation and contrast doses that patients will receive if they are to routinely undergo postoperative DSA.

\section{Conclusions}

With the largest series of which we are aware, to date, we provide additional evidence that ICG videoangiography is a useful adjunct in the surgical treatment of cranial dAVFs. ICG videoangiography is an appropriate primary modality with which to evaluate dAVFs intraoperatively

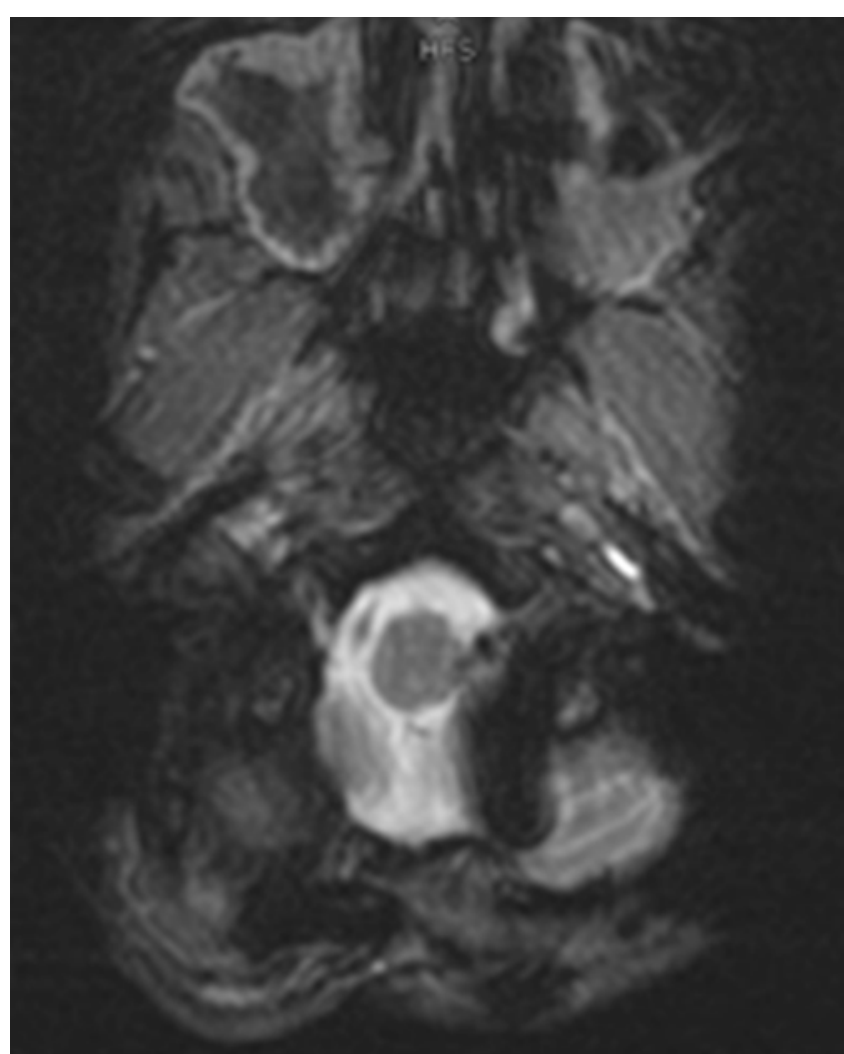

FIG. 8. Axial T2-weighted postoperative MR image showing resolution of edema within the cervical spinal cord.

and, in most cases, may serve as a replacement for intraoperative DSA for preobliteration planning and postligation confirmation of obliteration. However, a role for formal postoperative angiography remains regardless of the intraoperative modality used.

\section{References}

1. Beynon C, Herweh C, Rohde S, Unterberg AW, Sakowitz OW: Intraoperative indocyanine green angiography for microsurgical treatment of a craniocervical dural arteriovenous fistula. Clin Neurol Neurosurg 114:696-698, 2012

2. Cognard C, Casasco A, Toevi M, Houdart E, Chiras J, Merland JJ: Dural arteriovenous fistulas as a cause of intracranial hypertension due to impairment of cranial venous outflow. J Neurol Neurosurg Psychiatry 65:308-316, 1998

3. Collice M, D'Aliberti G, Arena O, Solaini C, Fontana RA, Talamonti G: Surgical treatment of intracranial dural arteriovenous fistulae: role of venous drainage. Neurosurgery 47:56-67, 2000

4. Derdeyn CP, Moran CJ, Cross DT, Grubb RL Jr, Dacey RG Jr: Intraoperative digital subtraction angiography: a review of 112 consecutive examinations. AJNR Am J Neuroradiol 16:307-318, 1995

5. Hänggi D, Etminan N, Steiger HJ: The impact of microscope-integrated intraoperative near-infrared indocyanine green videoangiography on surgery of arteriovenous malformations and dural arteriovenous fistulae. Neurosurgery 67:1094-1104, 2010

6. Hoh BL, Choudhri TF, Connolly ES Jr, Solomon RA: Surgical management of high-grade intracranial dural arteriovenous fistulas: leptomeningeal venous disruption without nidus excision. Neurosurgery 42:796-805, 1998 
7. Kato N, Tanaka T, Suzuki Y, Sakamoto H, Arai T, Hasegawa Y, et al: Multistage indocyanine green videoangiography for the convexity dural arteriovenous fistula with angiographically occult pial fistula. J Stroke Cerebrovasc Dis 21:918. e1-918.e5, 2012

8. Killory BD, Nakaji P, Gonzales LF, Ponce FA, Wait SD, Spetzler RF: Prospective evaluation of surgical microscopeintegrated intraoperative near-infrared indocyanine green angiography during cerebral arteriovenous malformation surgery. Neurosurgery 65:456-462, 2009

9. Killory BD, Nakaji P, Maughan PH, Wait SD, Spetzler RF: Evaluation of angiographically occult spinal dural arteriovenous fistulae with surgical microscope-integrated intraoperative near-infrared indocyanine green angiography: report of 3 cases. Neurosurgery 68:781-787, 2011

10. Newton TH, Cronqvist $\mathrm{S}$ : Involvement of dural arteries in intracranial arteriovenous malformations. Radiology 93:1071-1078, 1969

11. Raabe A, Beck J, Gerlach R, Zimmermann M, Seifert V: Near-infrared indocyanine green video angiography: a new method for intraoperative assessment of vascular flow. Neurosurgery 52:132-139, 2003

12. Raabe A, Beck J, Seifert V: Technique and image quality of intraoperative indocyanine green angiography during aneurysm surgery using surgical microscope integrated near-infrared video technology. Zentralbl Neurochir 66:1-8, 2005

13. Raabe A, Nakaji P, Beck J, Kim LJ, Hsu FP, Kamerman JD, et al: Prospective evaluation of surgical microscopeintegrated intraoperative near-infrared indocyanine green videoangiography during aneurysm surgery. J Neurosurg 103:982-989, 2005

14. Schuette AJ, Cawley CM, Barrow DL: Indocyanine green videoangiography in the management of dural arteriovenous fistulae. Neurosurgery 67:658-662, 2010

15. Woitzik J, Horn P, Vajkoczy P, Schmiedek P: Intraoperative control of extracranial-intracranial bypass patency by nearinfrared indocyanine green videoangiography. J Neurosurg 102:692-698, 2005

\section{Author Contributions}

Conception and design: all authors. Acquisition of data: Thind, Hardesty. Analysis and interpretation of data: Nakaji, Thind, Hardesty. Drafting the article: Nakaji, Thind, Hardesty. Critically revising the article: all authors. Reviewed submitted version of manuscript: all authors. Approved the final version of the manuscript on behalf of all authors: Nakaji. Statistical analysis: Hardesty. Administrative/technical/material support: Nakaji, Zabramski, Spetzler. Study supervision: Nakaji, Zabramski, Spetzler.

\section{Supplementary Information Current Affiliation}

Dr. Thind: Department of Neurosurgery, School of Medicine and Biomedical Sciences, State University of New York at Buffalo, NY.

\section{Correspondence}

Peter Nakaji, c/o Neuroscience Publications, Barrow Neurological Institute, St. Joseph's Hospital and Medical Center, $350 \mathrm{~W}$. Thomas Rd., Phoenix, AZ 85013. email: neuropub@dignity health.org. 